\title{
Enhanced absorption of hydroxysafflor yellow A using a self-double-emulsifying drug delivery system: in vitro and in vivo studies
}

This article was published in the following Dove Press journal:

International Journal of Nanomedicine

27 July 2012

Number of times this article has been viewed

\author{
Liang-Zhong Lv' \\ Chen-Qi Tong' \\ Qing $L v^{2,3}$ \\ Xin-Jiang Tang ${ }^{2}$ \\ Li-Ming $\mathrm{Li}^{2}$ \\ Qing-Xia Fang' \\ Jia Yu' \\ Min $\mathrm{Han}^{2}$ \\ Jian-Qing $\mathrm{Gao}^{2}$
}

'Department of Pharmacy, Zhejiang Provincial People's Hospital, 'Institute of Pharmaceutics, College of

Pharmaceutical Sciences, Zhejiang University, ${ }^{3}$ Pharmaceutics Laboratory of TCM, Zhejiang Traditional Chinese Medicine University, Hangzhou, China

Correspondence: Min Han Institute of Pharmaceutics, College of Pharmaceutical Sciences, Zhejiang University, Hangzhou 31 0058, China

Tel +86 57l 88208437

Fax +86 57। 88208437

Email hanmin2@zju.edu.cn
Abstract: Hydroxysafflor yellow A (HSYA), the main active ingredient of the safflower plant (Carthamus tinctorius L.), is a hydrophilic drug with low oral bioavailability. Water-in-oil-in-water $(\mathrm{w} / \mathrm{o} / \mathrm{w})$ double emulsions may enhance the oral absorption of HSYA. In this study, we prepared a selfdouble-emulsifying drug delivery system (SDEDDS) to improve the absorption of HSYA. SDEDDS consists of water in oil emulsions and hydrophilic surfactants that can self-emulsify into w/o/w double emulsions in the aqueous gastrointestinal environment. Confocal laser scanning micrographs showed that spherical droplets were uniformly distributed in the dispersion medium with narrow particle size distribution and could form fine w/o/w double emulsions upon dilution in dispersion medium with gentle stirring. The dispersed oil droplets contained small dispersed aqueous droplets consistent with the characteristics of double emulsions. Furthermore, in vitro cellular experiments were performed to study the mechanism of the absorption promoting effect of SDEDDS. The accumulation of rhodamine-123 in Caco-2 cells was used to evaluate the efflux transport of p-glycoprotein inhibitor. Histopathologic studies on the rat intestine showed that SDEDDS can cause mucosal damage to a certain degree of toxicity, however this was not serious. These results suggest that SDEDDS can greatly improve the oral absorption of HSYA. Given the toxicity demonstrated to the small intestine, the formulation prescription should be improved to enhance security in the future.

Keywords: self-double-emulsifying drug delivery system, hydroxysafflor yellow A, Caco-2 cells, bioavailability, histopathologic studies

\section{Introduction}

Hydroxysafflor yellow A (HSYA) is the main active ingredient of the safflower plant (Carthamus tinctorius L). A traditional Chinese drug, HSYA has been demonstrated to antagonize platelet activating factor receptor binding, and is used to treat several dysemia diseases such as myocardial ischemia, cerebral ischemia, coronary heart disease, and cerebral thrombosis. ${ }^{1-3}$ HSYA, according to the biopharmaceutics classification system (BCS) is a Class III drug that is hydrophilic, with very poor intestinal membrane permeability and low oral bioavailability. ${ }^{4}$

So far, only the injection of HSYA has been widely used in clinical therapy, ${ }^{5,6}$ the low oral bioavailability of BCS class III drugs deeming them unsuitable for oral formulations.

In the past, many methods including the addition of an absorption enhancer, ${ }^{4}$ chemical modification, ${ }^{7}$ and pharmaceutical means of drug delivery have been employed to solve these issues. From these studies, water-in-oil-in-water $(w / o / w)$ double emulsions show great potential for promoting the oral absorption of hydrophilic drugs. ${ }^{8,9}$ Water in oil in water double emulsions are complex liquid dispersion 
systems consisting of inner water, oil, and outer water phases, hydrophilic drugs loaded into the internal aqueous droplets. Thus, self-double-emulsifying drug delivery systems (SDEDDS) seem to be a suitable formulation for HSYA. A previous study has shown that this structure could improve the oral bioavailability of BCS Class III drugs to a certain extent. ${ }^{8}$ However, double emulsions are thermodynamically unstable with a strong tendency towards coalescence, flocculation, and creaming. ${ }^{10}$ Moreover, they are sensitive to heat, $\mathrm{pH}$ change, and organic solvents.

In this study, a SDEDDS was prepared to increase the oral absorption of HSYA. SDEDDS is composed of water in oil (w/o) emulsions and hydrophilic surfactants with drugs encapsulated in the inner water phase. It can spontaneously emulsify into w/o/w double emulsions in the presence of gastrointestinal peristaltic movements in the aqueous gastrointestinal environment, and is more stable compared with conventional thermodynamically unstable double emulsions. ${ }^{11}$ This novel formulation could markedly enhance the intestinal absorption of HSYA in vitro and in vivo.

\section{Materials and methods Reagents and chemicals}

HSYA (98\% purity) and tetramethylpyrazine (TMP) were supplied by the Chengdu Herb purity Company, Ltd (Chengdu, China). Medium-chain triglycerides, oleic acid and labrasol were obtained from Gattefosse (Saint-Priest, France). Bean phospholipids and gelatin were purchased from Lipoid (Ludwigshafen, Germany) and the Shanghai Zhaorui Biotech Company, Ltd (Shanghai, China), respectively. Tween ${ }^{\circledR} 80$ was obtained from the Wenzhou Qingming Chemical Plant (Wenzhou, China). Chlorpromazine was purchased from the Shanghai Harvest Pharmaceutical Company, Ltd (Shanghai, China). Methyl- $\beta$-cyclodextrin was obtained from the Shanghai Ding Jie Biological Technology Company, Ltd (Shanghai, China). $\mathrm{NaN}_{3}$, amiloride, and 3-(4,5-dimethylthiazol -2-yl)2,5-diphenyltetrazolium bromide (MTT) were purchased from Sigma-Aldrich (St Louis, MO). N-acetyl-L-cysteine and polyoxyethylene (10) octylphenyl ether (Triton X-100) were purchased from Sigma-Aldrich. Dulbecco's Modified Eagle Medium (DMEM) and fetal bovine serum were purchased from Thermo Fisher Biochemical Products (Beijing, China). The bicinchoninic acid protein assay kit was obtained from Nanjing Bi Yun Tian Kits Biotechnology Company (Jiangsu, Beijing, China). Sephadex G-100 was supplied by the Beijing Investor Science and Technology Development Company, Ltd (Beijing, China). All other chemicals were of analytical or chromatographic grade.

\section{Animals}

Male Sprague Dawley rats weighing $200 \mathrm{~g} \pm 20 \mathrm{~g}$ were obtained from the Animal Center of the Zhejiang University (Hangzhou, China) and housed under normal laboratory conditions of temperature, relative humidity, and light. The rats had free access to standard laboratory diet and water before the experiment. Animal experiments were approved by the Animal Experimental Ethical Committee of Zhejiang University, Hangzhou, China.

\section{Preparation of HSYA-SDEDDS}

SDEDDS was prepared through emulsification. HSYA (48 mg) was dissolved in $0.5 \%$ gelatin solution as the inner water phase. The oil phase contained bean phospholipids, medium chain triglycerides, Tween 80, oleic acid, and labrasol (20/65/7.4/2.5/0.1, in wt $\%$ ). The inner phase was added to the oil phase with moderate magnetic stirring $(400 \mathrm{rpm})$ at room temperature. The w/o emulsions were then homogenized at $9500 \mathrm{rpm}$ for 3 minutes (FJ-200 high-speed dispersion homogenizer; Jiangsujintan Jincheng Instruments Co, Jiangsu, China.) until a clear and transparent formulation was obtained.

\section{Characterization of SDEDDS formulations}

Droplet size analysis and microscopic observation

The morphology and droplet size distribution of the formulation after transformation into w/o/w emulsions were studied under an inverted fluorescence microscope (DM1400B; Leica, Sylvius, Germany) and dynamic light scattering (Nano-S90; Malvern Instruments Ltd, Worcestershire, United Kingdom.) respectively.

\section{Confocal laser scanning microscopy (CLSM)}

The freshly prepared SDEDDS was observed using CLSM (BX61W1-FV1000; Japan). SDEDDS containing $5 \mu \mathrm{g} / \mathrm{mL}$ sodium fluorescein was freshly prepared, dropped on an ordinary slide, and then covered with a cover slip. All the samples were detected using an $\mathrm{Ar} / \mathrm{Kr}$ laser with an excitation line of $491 \mathrm{~nm}$.

\section{Leakage of HSYA from the inner water phase}

The leakage of HSYA from the inner phase of the w/o/w double emulsions was measured through the Sephadex column method using Sephadex G-100. The prepared SDEDDS and distilled water (1:4) were mixed to form fine w/o/w double emulsions. A mixture of w/o/w emulsions and methanol (1:4) was centrifuged at 13,000 rpm for $5 \mathrm{~min}$ utes. The supernatant $\left(\mathrm{F}_{\text {total }}\right)$ was measured at $403 \mathrm{~nm}$ using an ultraviolet spectrophotometer (TU-1800 PC; Beijing, China). The same amount of w/o/w emulsion was treated in 
a Sephadex column. The emulsion $\left(\mathrm{F}_{\mathrm{in}}\right)$ that filtered down from the Sephadex column was treated and measured as described above.

The leakage of HSYA from the inner water phase of the transformed w/o/w emulsion was calculated using the following equation:

$$
\text { Leakage of } \operatorname{HSYA}(\%)=\left(1-\mathrm{F}_{\text {in }} / \mathrm{F}_{\text {out }}\right) \times 100
$$

\section{In vitro Caco-2 studies Cytotoxicity (MTT assay)}

Caco-2 cells were seeded in 96-well plates at a density of 10,000 cells/well and then incubated with culture medium for 24 hours before the experiments. At the beginning of the experiment, the culture medium was removed and replaced with $200 \mu \mathrm{L}$ of blank SDEDDS (of different concentrations) or the control (culture medium). After incubating the formulations for 2 hours, $20 \mu \mathrm{L}$ of MTT reagent $(5 \mathrm{mg} / \mathrm{mL})$ in phosphate buffered saline (PBS) was added to each well and the mixtures were incubated for 4 hours. After incubation, $180 \mu \mathrm{L}$ of dimethyl sulfoxide (DMSO) was added to each well. After complete dissolution of the crystals, absorbance values were measured at a wavelength of $570 \mathrm{~nm}$ using a KHB-ST-360 microplate reader (KHB, China). Cell viability was calculated using the following formula:

$$
\text { Cell viability }(\%)=\mathrm{A}_{\text {text }} / \mathrm{A}_{\text {control }} \times 100
$$

\section{Uptake studies of HSYA}

Caco- 2 cells were cultured at $37^{\circ} \mathrm{C}$ in DMEM supplemented with $10 \%$ fetal bovine serum. Six-well plates were used to seed the cells at a density of $5 \times 10^{4}$ cells $/ \mathrm{cm}^{2}$ for the uptake experiments. The cells were then cultured for 14 days before use. The uptake experiments were initiated by incubating the plates with different concentrations of HSYA solutions and HSYA-loaded SDEDDS for 2 hours. The cells were washed thrice with ice-cold PBS, frozen and thawed thrice, resuspended in water $(1 \mathrm{~mL})$, and then probe-sonicated 25 times $\left(4^{\circ} \mathrm{C}, 150\right.$ Watts, active every 2 seconds within a 3 second duration) to obtain cell lysates. The lysates were centrifuged at $15,000 \mathrm{rpm}$ for 10 minutes and the supernatant was analyzed through high-performance liquid chromatography (HPLC).

\section{Transport studies of HSYA}

The Caco- 2 cells were seeded onto a 12 -well polycarbonate membrane transwell with a pore size of $0.4 \mu \mathrm{m}$ and a growth surface area of $1.12 \mathrm{~cm}^{2}$ at a density of $5 \times 10^{4}$ cells $/ \mathrm{cm}^{2}$. Monolayers were used at 19 to 22 days after seeding. To ensure the integrity of each cell monolayer the transepithelial electrical resistance was checked. Transport studies were conducted by adding $0.4 \mathrm{mg} / \mathrm{mL}$ HSYA-SDEDDS and different concentrations of HSYA solutions to the apical cell side, and blank Hank's buffered salt solution to the basolateral cell side. Samples were collected from the basolateral side at predetermined intervals of $15,30,45,60,90$, and 120 minutes. HSYA concentrations were detected using HPLC. Apparent permeability $\left(\mathrm{P}_{\text {app }}\right)$ was calculated as follows:

$$
\mathrm{P}_{\mathrm{app}}=\frac{\mathrm{dQ}}{\mathrm{dt}} \times\left(\frac{1}{\mathrm{AC}_{0}}\right),
$$

where $\mathrm{dQ} / \mathrm{dt}$ is the permeability rate, $\mathrm{A}$ is the surface area of the monolayer, and $\mathrm{C}_{0}$ is the initial concentration of the drug.

\section{Cellular uptake study of rhodamine- 123 (R-I23)}

The Caco- 2 cells were seeded in a petri dish ( $35 \mathrm{~mm}$ diameter), added with blank SDEDDS diluted 50-fold, and then incubated for 48 hours before the experiment. The cells were added with $150 \mu \mathrm{g} / \mathrm{mL}$ of cyclosporin A (CsA, a recognized p-glycoprotein [p-gp]) and then incubated for 1 hour before the experiment to serve as the positive control. On the day of the experiment, the cells were exposed to $2.5 \mu \mathrm{g} / \mathrm{mL}$ of R-123 for 2 hours in the presence of SDEDDS and CsA in DMEM. The cells were washed thrice with ice-cold PBS and then treated with $4 \%$ paraformaldehyde for 20 minutes. The fluorescence of the accumulated R-123 was measured through CLSM, and the fluorescence intensity was added using the Metamorph offline 7.7.6 software (Olympus, Tokyo, Japan).

\section{Pharmacokinetic study}

The rats were fasted overnight before the intragastric (ig) administration. Six rats were divided randomly into two groups and treated (ig) with $5 \mathrm{mg} / \mathrm{kg}$ of HSYA solution $(5 \mathrm{mg} / \mathrm{mL})$ and HSYA-SDEDDS formulation $(5 \mathrm{mg} / \mathrm{mL})$, respectively. After oral administration, blood samples $(0.2 \mathrm{~mL})$ were collected from the eye ground vein at 5, 15, 30, 45, 60, 90, 120, 180, 240, 360,480 , and 1440 minutes. Each sample was immediately transferred to heparinized plastic centrifuge tubes and then centrifuged at 12,000 rpm for 5 minutes. To allow precipitation of proteins, $200 \mu \mathrm{L}$ of plasma and $100 \mu \mathrm{L}$ of $6 \%$ perchloric acid were mixed. All samples were vortexed for 2 minutes and then centrifuged at $12,000 \mathrm{rpm}$ for 10 minutes. For liquid chromatography, $50 \mu \mathrm{L}$ of the supernatant was injected.

The HSYA aqueous solution was used as the control to calculate the relative bioavailability $\left(\mathrm{F}_{\text {rel }}\right)$. The $\mathrm{F}_{\text {rel }}$ of the HSYA-SDEDDS was calculated as follows:

$$
\mathrm{F}_{\text {rel }}(\%)=\left(\mathrm{AUC}_{1} \times \mathrm{D}_{\text {control }}\right) /\left(\mathrm{AUC}_{\text {control }} \times \mathrm{D}_{1}\right) \times 100,
$$


where $\mathrm{AUC}_{1}$ and $\mathrm{AUC}_{\text {control }}$ are the areas under the concentration time curve of HSYA after administration of HSYA-SDEDDS and HSYA solution, respectively, and $\mathrm{D}_{\text {control }}$ and $\mathrm{D}_{1}$ are the dosages of HSYA after administration of HSYA-SDEDDS and HSYA solution, respectively.

\section{Histopathologic studies}

The rats were fasted for approximately 20 hours before the experiment. At 8 hours after administration of $5 \%$ glucose solution (control) and $1 \mathrm{~mL}$ of HSYA-SDEDDS formulation, a portion of the intestine from the middle of the small intestine was carefully excised and then washed with 5\% glucose solution. As a positive control, a mixture of $\mathrm{N}$-acetyl-1-cysteine and Triton X-100 was also administered to the rats according to the method of Takatsuka et al. ${ }^{12}$ All the dissected intestines were placed overnight in a fixative solution ( $4 \%$ paraformaldehyde) at $4{ }^{\circ} \mathrm{C}$. After removing the fixative, the intestine pieces were successively incubated in the following solutions: PBS for 30 minutes, 30\% ethanol for 1 hour, $70 \%$ ethanol for 1.5 hours, $80 \%$ ethanol for 2 hours, 95\% ethanol for 2 hours, and 100\% ethanol for 4 hours. Subsequently, paraffin sections were prepared and stained with hematoxylin-eosin and then examined using an inverted fluorescence microscope (DM1400B; Germany).

\section{HPLC analysis of samples}

Chromatographic conditions were performed according to the literature, ${ }^{13}$ with some modifications. Samples were determined using an Agilent 1200 HPLC system with ZORBAX SB-C18 (4.6 mm × $250 \mathrm{~mm}, 5 \mu \mathrm{m})$ (Agilent, Santa Clara, CA). The mobile phase for HSYA analysis was methanol: acetonitrile: $0.1 \%$ phosphate solution (32:2:66). The flow rate was $0.8 \mathrm{~mL} / \mathrm{min}$. The column oven temperature was $40^{\circ} \mathrm{C}$, and detection was performed at a wavelength of $403 \mathrm{~nm}$.

\section{Statistical analysis}

Results are expressed as mean \pm SD. Statistical comparisons were made using the two-tailed student's $t$-test and comparisons amongst more than three groups were performed using the one-way analysis of variance (ANOVA). $P<0.05$ was considered to be significant.

\section{Results}

\section{Characterization of SDEDDS formulations}

As shown in Figure 1, the freshly prepared HSYA-SDEDDS can formulate fine w/o/w double emulsions after 5-fold dilutions with water under gentle stirring at $37^{\circ} \mathrm{C}$ for

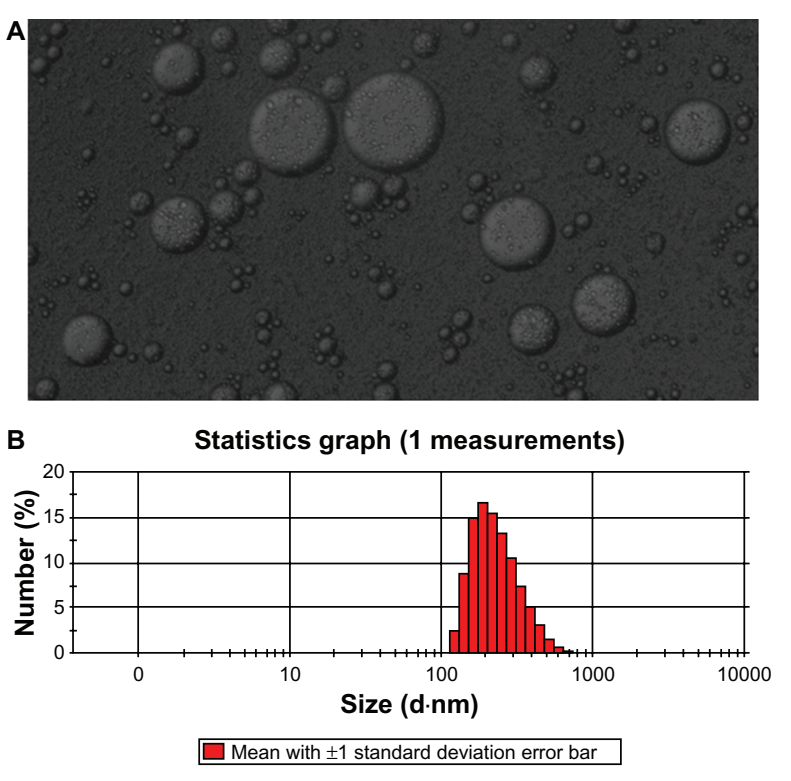

Figure I (A) Microscopy image of freshly prepared HSYA-SDEDDS transformed into w/o/w double emulsions after 2-minute dilution with dispersion medium. (B) Size distribution of the formulation after formation of fine w/o/w double emulsions.

Abbreviations: HSYA, Hydroxysafflor yellow A; SDEDDS, self-double-emulsifying drug delivery system; w/o/w, water-in-oil-in-water.

2 minutes. HSYA was encapsulated in the internal water phase of the double emulsions. Dynamic light scattering studies showed that the mean diameter of the transformed w/o/w double emulsions was approximately $277 \mathrm{~nm}$.

CLSM micrographs (Figure 2A) showed that the spherical droplets were uniformly distributed in the dispersion medium with narrow particle size distribution. As shown in Figure 2B, the dispersed oil droplets contained small dispersed aqueous droplets consistent with the characteristics of double emulsions. The leakage rate of SDEDDS after transformation into double emulsions was approximately $29.48 \%$.

\section{Cytotoxicity (MTT assay)}

As shown in Figure 3D, the blank SDEDDS diluted 10-, 15-, 30-, and 50-fold showed almost no cytotoxicity on Caco- 2 cells after incubation for 2 hours.
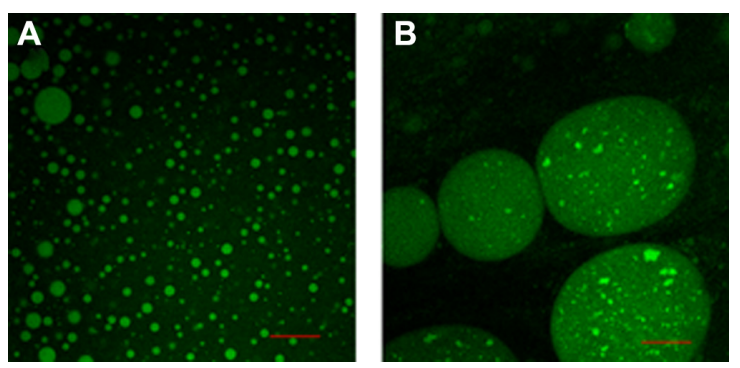

Figure 2 (A) Confocal microscopy images of freshly prepared sodium fluoresceinSDEDDS and (B) formulated fine w/o/w double emulsions after 2-minute dilution with dispersion medium.

Note: Scale bar represents $30 \mu \mathrm{m}$.

Abbreviation: SDEDDS; self-double-emulsifying drug delivery system; w/o/w, water-in-oil-in-water. 

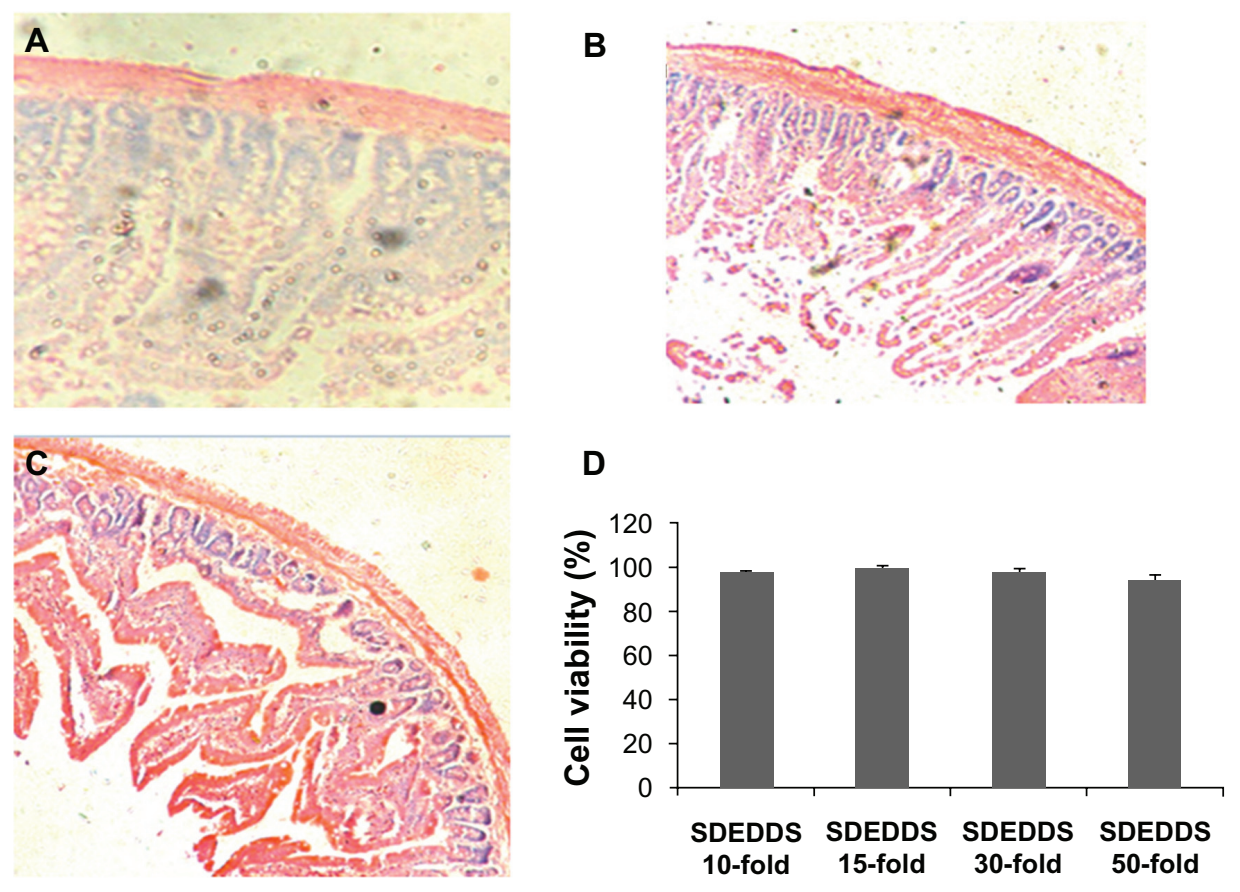

Figure 3 Histological sections of intestinal segments treated according to (A) control, (B) positive control conditions, and (C) blank SDEDDS (H\&E stain, $\times$ I00). (D) Effect of blank SDEDDS on the cytotoxicity of Caco-2 cells after incubation for 2 hours.

Note: Formulation was removed, and culture was continued up to 48 hours.

Abbreviations: H\&E, hematoxylin and eosin; HSYA, Hydroxysafflor yellow A; SDEDDS, self-double-emulsifying drug delivery system.

\section{Uptake and transport studies of HSYA}

As shown in Figure 4A and B, the cellular uptake of HSYA rose linearly with increasing concentration after incubation with different concentrations of HSYA solutions at $37^{\circ} \mathrm{C}$ for 2 hours. This result indicates that HSYA is absorbed mainly through passive diffusion. Results of the HSYA uptake and transport experiments confirmed that the membrane permeability of HSYA is very low. In addition, SDEDDS significantly increased the permeability of HSYA across Caco-2 cell monolayers regardless of the drug concentration (Figure $4 \mathrm{C}$ and D). The Papp of $0.4 \mathrm{mg} / \mathrm{mL}$ HSYA was $(3.52 \pm 1.41) \times 10^{-6}$ $\mathrm{cm} / \mathrm{s}$ and then increased to $(6.62 \pm 2.61) \times 10^{-6} \mathrm{~cm} / \mathrm{s}$ when the same concentration of HSYA-SDEDDS was applied to the Caco-2 cells. Figure 5A shows that TMP and CsA significantly increased the absorption of HSYA on Caco-2 cells.

To illustrate the mechanism underlying the enhancing effect of SDEDDS, endocytosis inhibitor studies were conducted (Figure 5B). Cells were pretreated for 30 minutes with various inhibitors, such as $\mathrm{NaN}_{3}$ $(1.32 \mathrm{mg} / \mathrm{mL})$ to inhibit mitochondrion, chlorpromazine $(10 \mu \mathrm{g} / \mathrm{mL})$ to inhibit clathrin vesicles, and methyl- $\beta-\mathrm{CD}$ $(13.3 \mathrm{mg} / \mathrm{mL})$ to inhibit caveolae, and amiloride $(50 \mu \mathrm{M})$ to inhibit pinocytosis. The uptake of HSYA-SDEDDS was $2.70 \pm 1.22 \mu \mathrm{g} / \mathrm{mg}$ protein. However, after 30 minutes of incubation with four kinds of inhibitors, the results yielded $4.06 \pm 1.19,3.14 \pm 1.39,2.97 \pm 0.37$, and $1.38 \pm 0.16 \mu \mathrm{g} / \mathrm{mg}$ protein. Compared with the control, the inhibitors did not show any significant effects on the uptake of HSYA.

\section{R-I 23 accumulation in Caco- 2 cells}

The accumulation of R-123 in Caco-2 cells was used to evaluate the efflux transport of p-gp (Figure 6). Compared with the R-123 solution group, the fluorescence intensity of the SDEDDS (preincubated for 48 hours) and CsA (preincubated for 1 hour) groups increased 14- and 21-fold (Figure 6B and C), respectively. These results indicate that blank SDEDDS preincubated for a period of time can inhibit the p-gp efflux of Caco-2 cells to some extent.

\section{Pharmacokinetic study}

The HSYA plasma concentration time curve after ig administration of HSYA solution and HSYA-SDEDDS to the Sprague Dawley rats is shown in Figure 7. The area under the concentration time curve of HSYA was significantly improved after oral administration of HSYA-SDEDDS. The main pharmacokinetic parameters are listed in Table 1. The relative bioavailability of HSYA after administration of SDEDDS to the rats was $217 \%$. The enhancing rate (2.17-fold) indicates that SDEDDS enhanced the intestinal absorption of HSYA. 
A

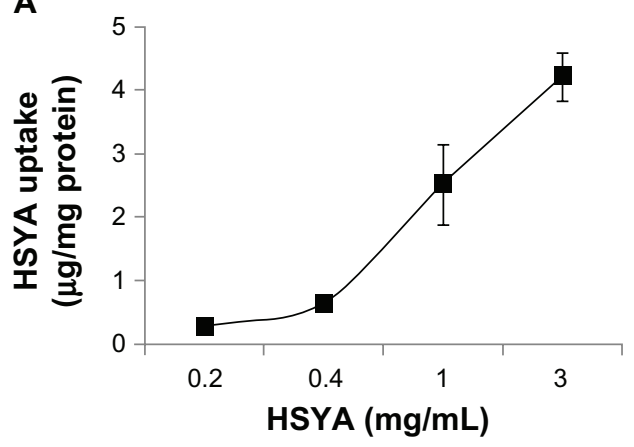

C

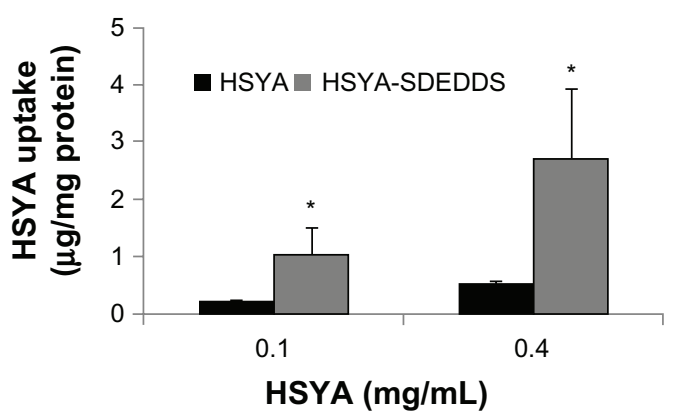

B

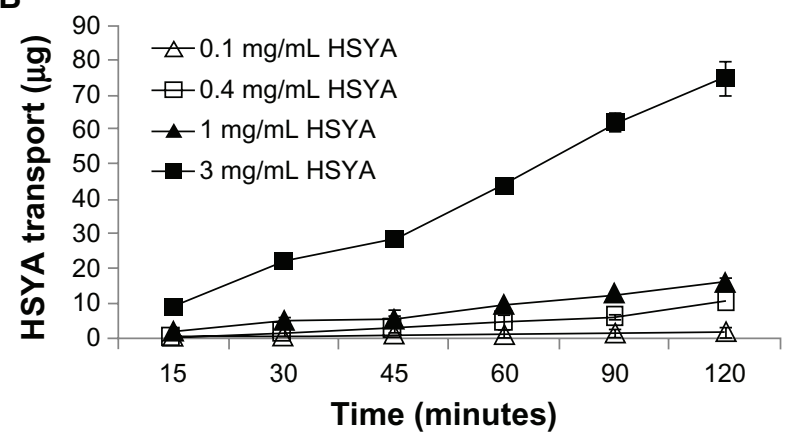

D

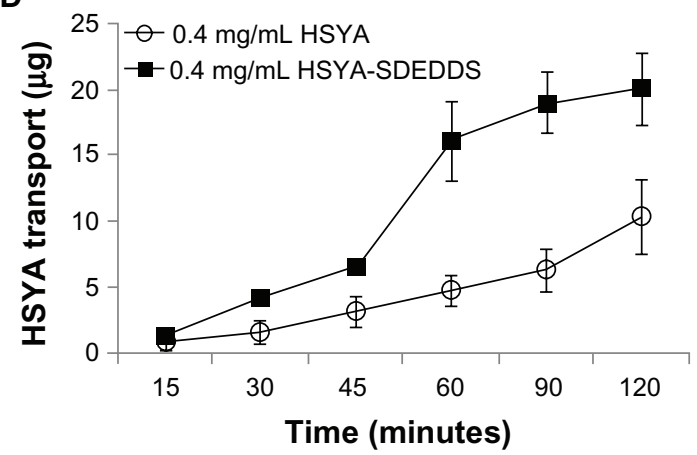

Figure 4 (A) Effect of different concentrations on HSYA uptake by Caco-2 monolayers after incubation with HSYA solutions for 2 hours. (B) Cumulative transport of HSYA across Caco-2 cell monolayers (surface area of monolayer $=1.12 \mathrm{~cm} 2$ ). (C) Effect of SDEDDS on Caco-2 cellular uptake. (D) Effect of SDEDDS on Caco-2 cellular transport (surface area of monolayer $=1.12 \mathrm{~cm} 2$ ).

Note: $* \mathrm{P}<0.05$, compared with control.

Abbreviations: HSYA, Hydroxysafflor yellow A; SDEDDS, self-double-emulsifying drug delivery system

\section{Toxicity of SDEDDS to rat intestines}

Absorption enhancers (eg, surfactants) improve intestinal absorption; however, they have also been reported to cause intestinal mucosal injury. ${ }^{14}$ Thus, we used histopathologic analysis to evaluate the extent of small intestine damage. Figure $3 \mathrm{~A}$ and $\mathrm{C}$ illustrate images of the intestinal segments 8 hours after ig administration of $5 \%$ glucose solution (control) and HSYA-SDEDDS. As a positive control of mucosal damage, the image of the intestinal segment 2 hours after administration of the solution containing N-acetyl-1-cysteine and Triton X-100 revealed a decidual alteration in the epithelial membranes (Figure 3B). As shown in Figure 3C, the top of the villi was lost, and the villus height became shortened. This result suggests that the SDEDDS formulation can cause mucosal damage to a certain extent. Figure 3D shows that the blank SDEDDS
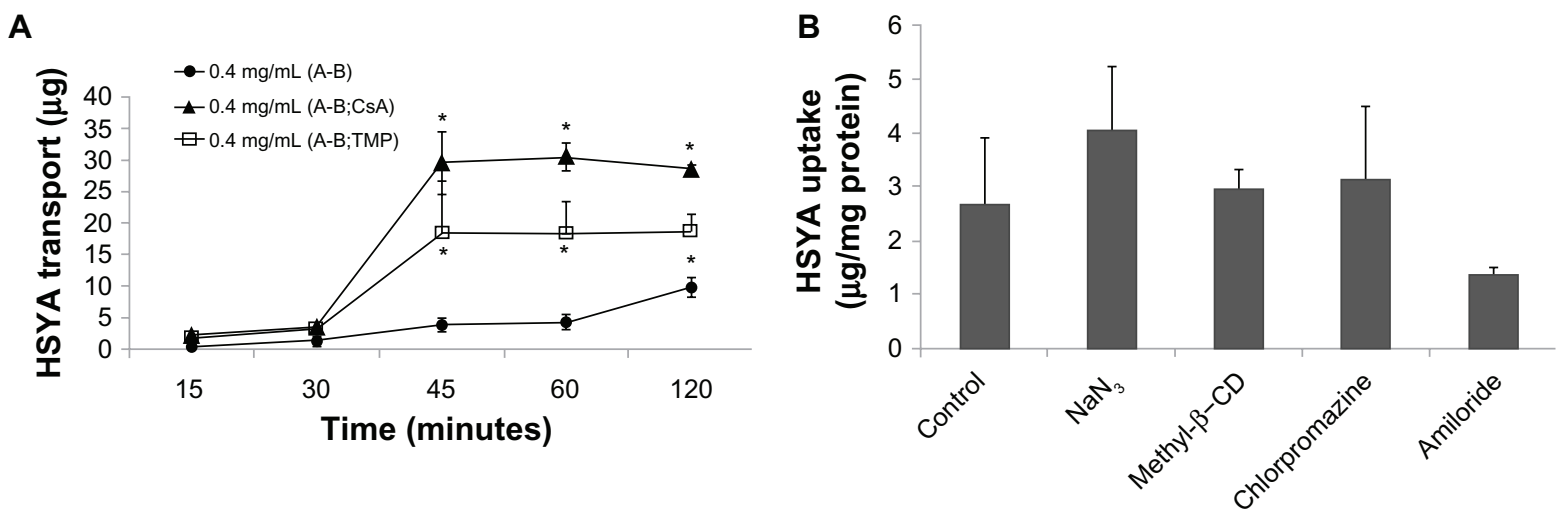

Figure 5 (A) Effect of p-gp inhibitors (cyclosporin A and tetramethylpyrazine) on cellular transport of HSYA solution. (B) Endocytosis inhibitor studies on Caco-2 cells of HSYA-SDEDDS with three kinds of endocytosis inhibitors and NaN3 to inhibit mitochondrion.

Note: ${ }^{*} P<0.05$, compared with control.

Abbreviations: HSYA, Hydroxysafflor yellow A; p-gp, p-glycoprotein; SDEDDS, self-double-emulsifying drug delivery system. 


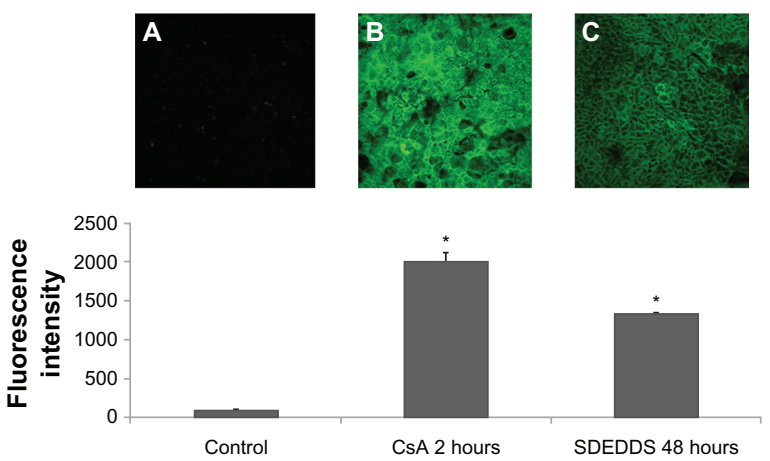

Figure 6 Rhodamine- 123 accumulation in Caco-2 cells. (A) cells without treatment; (B) cells preincubated with CsA for 2 hours; and (C) cells preincubated with SDEDDS for 48 hours.

Note: ${ }^{*} P<0.05$, compared with control.

Abbreviations: CsA, cyclosporin A; SDEDDS, self-double-emulsifying drug delivery system.

diluted 10-, 15-, 30-, and 50-fold with dispersion medium did not elicit any cytotoxic effect on the Caco-2 cells after 2 hours incubation, followed by removal of the formulation to continue the culture for 48 hours at $37^{\circ} \mathrm{C}$.

\section{Discussion}

HSYA, the main active ingredient of the safflower plant (Carthamus tinctorius L.), is widely used in the clinical treatment of dysemia diseases such as myocardial ischemia, cerebral ischemia, coronary heart disease, and cerebral thrombosis. ${ }^{1,3}$ HSYA is a hydrophilic drug with low oral bioavailability belonging to the BCS III class of drugs. ${ }^{4}$ The results in Figure 4A and B confirm this fact. As a result, only the injection of HSYA has been widely used in clinical therapy. ${ }^{5,6}$ To improve its oral bioavailability, we developed a SDEDDS by formulating mixtures of w/o emulsion and hydrophilic surfactants. It can spontaneously emulsify into w/o/w double emulsions with the gastrointestinal peristaltic movements in the aqueous gastrointestinal environment, with drugs encapsulated in the internal water phase. Compared with conventional thermodynamically unstable double emulsions, SDEDDS is a stable formulation system ${ }^{11}$ and is more convenient for storage. As shown in Figures 1A and $2 \mathrm{~B}$, SDEDDS formed fine $\mathrm{w} / \mathrm{o} / \mathrm{w}$ double emulsions upon dilution with the dispersion medium under gentle stirring. The dispersed oil droplets contained small dispersed aqueous droplets consistent with the characteristics of double emulsions.

Results of the uptake and transport studies on the Caco-2 cells showed that SDEDDS remarkably increased the membrane permeability of HSYA across Caco-2 cell monolayers. The plasma concentration time profiles of HSYA-SDEDDS from pharmacokinetic studies in rats showed a 2.17-fold increase compared with the HSYA solution. We postulated that HSYA-SDEDDS is transported across the Caco-2 cells through endocytosis to improve the membrane permeability of HSYA. However, the endocytosis inhibitors (Figure 5B) did not show any significant effects on the uptake of HSYA. Based on the endocytosis studies, we assume that the transport of SDEDDS across the Caco-2 cell monolayers may not be through endocytosis but through passive diffusion or through the release of HSYA outside the cells, which is then transported into cells. p-gp inhibitors studies (Figure 5A) showed that TMP and CsA significantly increased the absorption of HSYA on Caco-2 cells. Hence, we confirmed that HSYA efflux could be a result of p-gp transport.

We conjectured that the formulations were possibly through the inhibition of the p-gp efflux to promote the absorption of HSYA. As shown in the R-123 experiments (Figure 6), the positive control group (CsA) and the blankSDEDDS significantly increased p-gp substrate R-123 absorption after preincubation. This result suggests that the

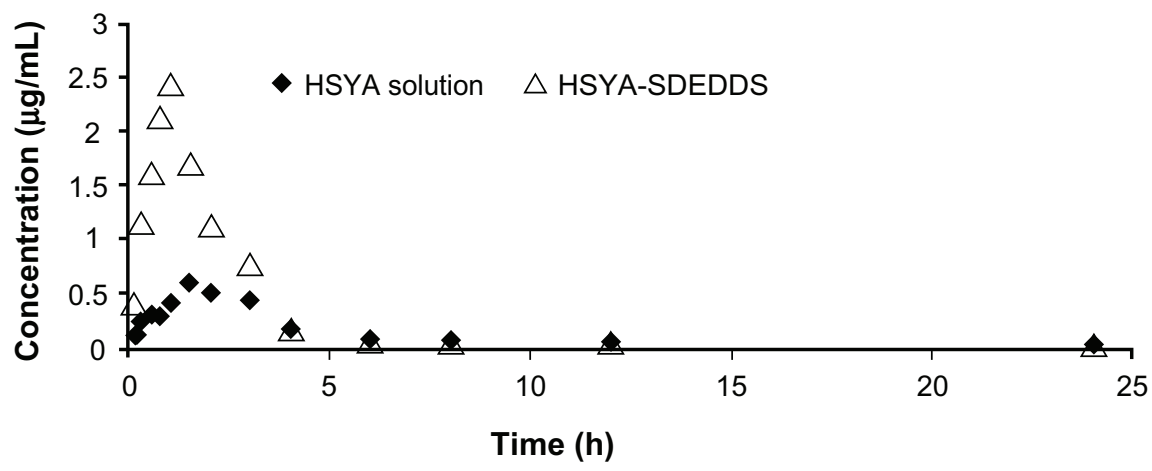

Figure 7 Plasma concentration time profiles of HSYA after intragastric administration of HSYA solution and HSYA-SDEDDS to Sprague Dawley rats. Abbreviations: HSYA, Hydroxysafflor yellow A; SDEDDS, self-double-emulsifying drug delivery system. 
Table I Pharmacokinetic parameters of HSYA after oral administration of HSYA-SDEDDS and HSYA solution

\begin{tabular}{llclll}
\hline & $\mathbf{C}_{\max }(\mu \mathrm{g} / \mathrm{ml})$ & $\mathbf{T}_{\max }(\mathbf{m i n})$ & AUC $_{\mathbf{0 - 1 2}}(\mu \mathrm{g} \mathrm{min} / \mathrm{ml})$ & MRT $(\mathbf{m i n})$ & $\mathbf{F}_{\text {rel }}(\%)$ \\
\hline Control & $0.64 \pm 0.14$ & $130 \pm 37.42$ & $156.81 \pm 9.53$ & $363.37 \pm 20.63$ & 100 \\
SDEDDS & $2.51 \pm 22.31$ & $55 \pm 8.66 *$ & $340.51 \pm 316.99$ & $279.51 \pm 110.87$ & 217 \\
\hline
\end{tabular}

Notes: All values reported are means $\pm S D(n=3)$. $* P<0.05$, compared with control.

Abbreviations: AUC, areas under the concentration time curve; HSYA, Hydroxysafflor yellow A; MRT, mean retention time; SD, standard deviation; SDEDDS, self-doubleemulsifying drug delivery system.

formulations can significantly inhibit p-gp efflux, which may be one of the reasons for the improvement of HSYA oral bioavailability in vivo. Some excipients of SDEDDS, such as Tween 80 and Labrasol (Gattefosse, Saint-Priest, France), have been reported to inhibit p-gp and thus potentially enhance drug absorption. ${ }^{14-16}$ The Papp of HSYA was $(3.52 \pm 1.41) \times 10^{-6} \mathrm{~cm} / \mathrm{s}$ and was improved by 1.88 -fold in the presence of SDEDDS. We assume that the enhancement of HSYA in vitro transport is mainly attributed to the formulations that inhibit p-gp function and increase membrane fluidity. The phospholipids in SDEDDS could also increase cell membrane fluidity. Tight junctions between cells may be opened at this condition, resulting in the enhanced permeability of hydrophilic drugs. ${ }^{17,18}$ Histopathologic studies (Figure 3C) confirmed that the prominent absorption-promoting effect of SDEDDS causes no serious local damage to the small intestinal mucosa. As figure 3D shows, blank SDEDDS incubated for 2 hours and then was removed, and culture was continued up to 48 hours, the cell viability was very high after that. This result indicates that toxicity of the preparation is reversible and will not affect the uptake and transport studies on Caco- 2 cells.

\section{Conclusion}

SDEDDS improved the absorption of HSYA mainly through inhibition of p-gp expression and Papp improvement of the water-soluble drug. SDEDDS showed no significant toxicity in vitro and in vivo. Our study has demonstrated that SDEDDS could be a promising technique for improving the oral absorption of drugs with high solubility and low permeability.

\section{Acknowledgment}

This study was supported by the Zhejiang Provincial Natural Science Foundation of China (Y2100564) and National Basic Research Program of China (2009CB930300).

\section{Disclosure}

The authors report no conflicts of interest in this work.

\section{References}

1. Zhu H, Wang Z, Ma C, et al. Neuroprotective effects of hydroxysafflor yellow A: in vivo and in vitro studies. Planta Med. 2003;69(5):429-433.
2. Zhu HB, Wang ZH, Tian JW, Fu FH, Liu K, Li CL. Protective effect of hydroxysafflor yellow A on experimental cerebral ischemia in rats. Yaо Xие Хие Bao. 2005;40(12):1144-1146.

3. Liu YN, Zhou ZM, Chen P. Evidence that hydroxysafflor yellow A protects the heart against ischaemia-reperfusion injury by inhibiting mitochondrial permeability transition pore opening. Clin Exp Pharmacol Physiol. 2008;35(2):211-216.

4. Wang S, Sun M, Ping Q. Enhancing effect of Labrafac Lipophile WL 1349 on oral bioavailability of hydroxysafflor yellow A in rats. Int J Pharm. 2008;358(1-2):198-204.

5. Qin F, Huang X. Guanxin II (II) for the management of coronary heart disease. Chin J Integr Med. 2009;15(6):472-476.

6. Liu SX, Zhang Y, Wang YF, et al. Upregulation of heme oxygenase-1 expression by hydroxysafflor yellow A conferring protection from anoxia/reoxygenation-induced apoptosis in $\mathrm{H} 9 \mathrm{c} 2$ cardiomyocytes. Int J Cardiol. April 14, 2011. [Epub ahead of print.]

7. Wang J, Chow D, Heiati H, Shen WC. Reversible lipidization for the oral delivery of salmon calcitonin. $J$ Control Release. 2003;88(3): 369-380.

8. Koga K, Takarada N, Takada K. Nano-sized water-in-oil-in-water emulsion enhances intestinal absorption of calcein, a high solubility and low permeability compound. Eur J Pharm Biopharm. 2010;74(2): 223-232.

9. Qi X, Wang L, Zhu J. Water-in-oil-in-water double emulsions: an excellent delivery system for improving the oral bioavailability of pidotimod in rats. J Pharm Sci. 2011;100(6):2203-2211.

10. Lutz R, Aserin A, Wicker L, Garti N. Release of electrolytes from W/O/W double emulsions stabilized by a soluble complex of modified pectin and whey protein isolate. Colloids Surf B Biointerfaces. 2009; 74(1):178-185.

11. Qi X, Wang L, Zhu J, Hu Z, Zhang J. Self-double-emulsifying drug delivery system (SDEDDS): a new way for oral delivery of drugs with high solubility and low permeability. Int J Pharm. 2011;409(1-2): 245-251.

12. Takatsuka S, Morita T, Koguchi A, Horikiri Y, Yamahara H, Yoshino H. Synergistic absorption enhancement of salmon calcitonin and reversible mucosal injury by applying a mucolytic agent and a non-ionic surfactant. Int J Pharm. 2006;316(1-2):124-130.

13. Tian Y, Yang ZF, Li Y, et al. Pharmacokinetic comparisons of hydroxysafflower yellow A in normal and blood stasis syndrome rats. $J$ Ethnopharmacol. 2010;129(1):1-4.

14. Cornaire G, Woodley J, Hermann P, Cloarec A, Arellano C, Houin G. Impact of excipients on the absorption of p-glycoprotein substrates in vitro and in vivo. Int J Pharm. 2004;278(1):119-131.

15. Lin Y, Shen Q, Katsumi H, et al. Effects of Labrasol and other pharmaceutical excipients on the intestinal transport and absorption of rhodamine123, a p-glycoprotein substrate, in rats. Biol Pharm Bull. 2007;30(7):1301-1307.

16. Rege BD, Kao JP, Polli JE. Effects of nonionic surfactants on membrane transporters in Caco-2 cell monolayers. Eur J Pharm Sci. 2002;16(4-5):237-246.

17. Sha X, Yan G, Wu Y, Li J, Fang X. Effect of self-microemulsifying drug delivery systems containing Labrasol on tight junctions in Caco-2 cells. Eur J Pharm Sci. 2005;24(5):477-486.

18. Yeh PY, Smith PL, Ellens H. Effect of medium-chain glycerides on physiological properties of rabbit intestinal epithelium in vitro. Pharm Res. 1994;11(8):1148-1154. 
International Journal of Nanomedicine

Dovepress

\section{Publish your work in this journal}

The International Journal of Nanomedicine is an international, peerreviewed journal focusing on the application of nanotechnology in diagnostics, therapeutics, and drug delivery systems throughou the biomedical field. This journal is indexed on PubMed Central, MedLine, CAS, SciSearch ${ }^{\circledR}$, Current Contents ${ }^{\circledR} /$ Clinical Medicine,
Journal Citation Reports/Science Edition, EMBase, Scopus and the Elsevier Bibliographic databases. The manuscript management system is completely online and includes a very quick and fair peer-review system, which is all easy to use. Visit http://www.dovepress.com/ testimonials.php to read real quotes from published authors.

Submit your manuscript here: http://www.dovepress.com/international-journal-of-nanomedicine-journal 